\title{
Posibilidades de Wikipedia en la docencia universitaria: elaboración colaborativa de conocimiento
}

\author{
Possibilities of Wikipedia in higher education: collaborative knowledge construction
}

\begin{abstract}
Tomás SAorín Pérez (1), María Verónica de HaRo y de SAN MATeo (2), Juan Antonio PASTOR SÁNCHEZ (3)
\end{abstract}

(1) Facultad de Comunicación y Documentación, Universidad de Murcia, tsp@um.es

(2) mvdeharo@um.es (3) pastor@um.es

\begin{abstract}
Resumen
Se presenta una guía para la incorporación del trabajo directo en la enciclopedia colaborativa Wikipedia como recurso didáctico en la docencia universitaria. Mientras que el uso de wikis en el aula está ampliamente documentado, las posibilidades que ofrece introducir a los alumnos en el proceso de edición de artículos de Wikipedia no lo están tanto. Se ofrece una clasificación de actividades susceptibles de ser realizadas por los alumnos, en el marco del desarrollo de los contenidos curriculares de las asignaturas, usando metodologías participativas. Uno de los aspectos más relevantes es la transformación del discurso crítico y desconfiado hacia la Wikipedia, en un conocimiento directo de su alcance, proceso de producción y sistemas de control de calidad, que actúa además como oportunidad de mejora real de una fuente de información usada recurrentemente por los alumnos universitarios, incidiendo en un contexto real de uso crítico y activo de las fuentes de información.

Palabras clave: Alfabetización informacional. Wikipedia. Estrategias didácticas participativas. Técnicas de investigación. Elaboración de trabajos científicos.
\end{abstract}

\section{Introducción}

La transformación tecnológica que han vivido los entornos de enseñanza y aprendizaje no afecta únicamente a aspectos materiales de la organización docente y al formato de los materiales utilizados. Uno de los aspectos que se trata de desarrollar a lo largo de la educación superior es la alfabetización informacional, la cual muchas veces queda reducida casi exclusivamente a la explotación de medios digitales como recurso didáctico y herramienta de productividad (comunicación, evaluación, publicación, etc.). Pero más allá de los campus virtuales, el medio digital transforma los roles del circuito de creación y difusión de información, no únicamente el formato y la capacidad de acceso. La publicación escolar está sometida a grandes corrientes de cambio, donde confluyen

\begin{abstract}
A guide for Wikipedia student edition as a collaborative active learning activity is presented. Whereas the use of wikis in the classroom is widely documented, the educational possibilities of Wikipedia itself are not so much. We offer a classification of participatory activities suitable for being carried out by the students in the development of the curricular contents. One of the most relevant aspects is the transformation of the critical and distrustful speech towards the Wikipedia in a direct knowledge of its scope, process of production and systems of quality control. In addition, it is a good opportunity to improve a widespread source of information among university undergraduates that has a real impact and for the students to develop a more critical and active use of information sources.
\end{abstract}

Keywords: Information literacy. Wikipedia. Active learning strategies. Research methods. Production of scientific works.

editoriales consolidadas, manuales de amplia difusión, regionalización de la edición, materiales de descarga limitada o cursos en línea con licencia Creative Commons.

Esta circunstancia nos plantea no sólo el reto de cómo usar las estrategias adecuadas para conseguir un aprendizaje autónomo y crítico de los contenidos específicos de las materias, sino también el de introducir a los alumnos, conforme avanzan en su ciclo formativo, en el sistema de producción de nuevos conocimientos, a una escala adecuada a su nivel académico y dedicación. Lo que convencionalmente se ha denominado "escritura científica" o "introducción a la metodología de investigación científica" ahora se ramifica en un amplio abanico de registros de comunicación digital, hacia los cuales también hemos de dirigirnos. 
Existen numerosas evidencias de que Wikipedia es una fuente de información usada de forma intensiva en el transcurso de la enseñanza superior como recurso para definir y localizar conceptos básicos y su contexto. Sin embargo tras su concepción utilitaria existe un enorme potencial para convertir Wikipedia en un laboratorio de trabajo intelectual, puesto que combina el sentido tradicional de elaboración de textos de síntesis conforme a unas pautas editoriales, con los nuevos requisitos de contenido abierto y supervisión colectiva.

Wikipedia es además un reto para el mundo académico español porque constituye una extraordinaria oportunidad de aumentar el potencial digital global de la sociedad. Es un indicador de un tipo de actividad digital que exige un mayor compromiso intelectual que las redes sociales al uso y casi diríamos que se convierte en el sustrato para el resto de los sectores de contenido digital, puesto que estimula la interrelación de fuentes, la precisión de los datos y la capacidad de movilizar una parte de las capacidades de una sociedad para generar un conocimiento libre, que apoye la base social para una sociedad más rica en conocimiento (Barberà, 2009). Podríamos preguntarnos por qué la edición alemana de Wikipedia es más extensa y de mejor calidad media que la española, estableciendo interesantes correlaciones entre datos demográficos y prácticas culturales.

A lo largo del resto del texto pasaremos rápidamente por los aspectos relacionados con la web social, la inteligencia colectiva o los cambios metodológicos introducidos por el Espacio Europeo de Educación Superior, sobre los que ya existe una abundante bibliografía. Centraremos el marco conceptual por un lado en la especificidad del modelo wiki de colaboración, y por otro en la naturaleza de las fuentes de información secundarias y terciarias de referencia, para desde allí sintetizar el modelo de relación entre las universidades y la comunidad Wikipedia, así como elementos prácticos para la realización de tareas en artículos y contenidos de la enciclopedia.

\section{Relevancia de Wikipedia: calidad, fiabilidad e indicador cultural}

El sitio web de Wikipedia lleva años posicionado entre los que mayor tráfico reciben. Es una web de construcción social en donde la interacción se plasma en textos informativos sobre todos los aspectos de la cultura. Se trata de una enciclopedia universal, en contacto directo con la actualidad, cuyo éxito la ha convertido en la mayor obra de referencia jamás publicada. En la categoría de contenidos finales, su alto posicionamiento en los buscadores ha incrementado su visibilidad, convirtiéndola en la fuente inicial de todo tipo de actividades de búsqueda de información en el ámbito de la cultura informal, la educación secundaria y la educación superior (Broughton, 2008).

La información digital no es ya simplemente una alternativa o una promesa de funcionalidad avanzada. Se constata una creciente preferencia, no sólo generacional, hacia el uso y reutilización de recursos informativos en línea, inmediatos y gratuitos, quedando postergadas otras fuentes de referencia clásicas de gran calidad y tradición. La existencia en línea y la gratuidad son los dos motivos determinantes en las preferencias de elección, a los que cabe sumar la familiaridad con el modelo editorial del artículo monográfico de carácter enciclopédico, expresado en lenguaje enunciativo y basado en referencias externas. La misma acumulación de contenidos y usuarios genera un ciclo de cuasimonopolio natural de unas fuentes sobre otras. En el caso de Wikipedia, el resto de enciclopedias se han ido desvaneciendo a su alrededor, dominando el sistema digital y aglutinando los esfuerzos de miles de colaboradores, cuyo poder confirman continuamente millones de usuarios (Lih, 2009).

Head y Eisenberg (2010) observan que Wikipedia es una fuente usada en el $85 \%$ de los trabajos de curso de los estudiantes universitarios y en el $91 \%$ de sus búsquedas relacionadas con problemas de la vida cotidiana.

Cuando hablamos de Wikipedia debemos precisar entre sus versiones, puesto que la versión en inglés, alemán, francés o español son espacios informativos independientes con excepción de las imágenes del Banco de Imágenes Wikimedia Commons (1). Wikipedia en español es un reflejo de la intensidad de la actividad digital en el ámbito cultural español e iberoamericano. La relación entre población hablante y la cantidad y calidad de contenidos en Wikipedia es un indicador derivado de nivel educativo, acceso a las redes o participación digital de una comunidad cultural. Que la edición en español sea más reducida que otras, pone en relación los datos brutos de población con el resto de elementos que construyen una sociedad del conocimiento (Gómez Hernández y Saorín Pérez, 2011).

El dominio del inglés como idioma de la ciencia, podría verse matizado desde la vitalidad de otros idiomas en su ámbito histórico-cultural propio. No puede considerarse a la versión española una versión de wikipedia local, sino que abarca la totalidad de los conocimientos científi- 
co-culturales. Por lo tanto sería razonable que tuviera una vitalidad pareja a la de sus industrias culturales, su producción científica y sus medios de comunicación social.

Aunque el acceso a los medios y fuentes de información socioculturales está cada vez más abierto, la naturaleza del trabajo enciclopedista se alimenta de una formación superior. ¿Cómo es la cultura informacional del colectivo universitario español de alumnos, profesores, investigadores y postgraduados? ¿Ha conectado ya con los nuevos modelos de generar conocimiento abierto de forma compartida o está aún dominada por los canales de publicación tradicional? ¿La digitalización masiva ha ido acompañada de un cambio cultural hacia la autoría colectiva y el dominio público? No hay una respuesta única para estas cuestiones, pero sí podemos afirmar que desde el estudio de Wikipedia pueden observarse tendencias en este sentido. La cadena clásica de producción y difusión de la ciencia y la cultura desde las universidades y otras instituciones culturales tiende a abrirse a la participación de una parte de la sociedad formada, activa, crítica, contracultural, una ciudadanía digital que añade un elemento clave en el consumo y creación de los referentes culturales. Si la publicación ha estado restringida a un núcleo de poder institucional, la cada vez mayor permeabilidad entre la cultura de masas y la cultura convencional, hace que sea necesaria una mayor participación del mundo de la academia en los medios sociales.

Pese a que la monografía titulada La revolución Wikipedia adopta un tono escéptico sobre el fenómeno, concluye aceptando que es necesario convivir con él (Assouline, 2008, p. 12):

Ocurre con esta enciclopedia como con todos los fenómenos sociales arraigados en las mentalidades desde su nacimiento: como existe, lo utilizamos. En lugar de ignorar la herramienta, tratemos de dominarla ejerciendo nuestra función crítica de contrapoder. [...] Y como el fenómeno seguramente está llamado a perdurar y a desarrollarse, porque sus principios (interactividad, rapidez, carácter gratuito, adaptabilidad) concuerdan con lo que está de moda, la gente se amolda. Lo que en mi caso significa, no consultarla ni utilizarla (nunca incluyo ningún vínculo a Wikipedia, hay que ser coherente), pero sí inducir a la vigilancia a los internautas creyentes y practicantes y, en particular entre éstos, a los estudiantes de escuelas, institutos y universidades.

La naturaleza participativa y la escasa presencia de jerarquía en los sistemas de revisión de artículos colisionan, en muchos sentidos, tanto con la tradicional fuente de autoridad académica, como con la polémica entre corrientes y escuelas de pensamiento. La política de neutra- lidad de la enciclopedia —ue trata de reflejar de forma proporcionada y distanciada todas las corrientes sobre un tema- difiere del criterio editorial de selección por calidad, fiabilidad y coherencia.

A la luz de esta situación podríamos volver a contemplar las reflexiones del propio Diderot como declaraciones de intenciones de un proyecto en continua construcción cuando planteaba los muchos problemas de calidad de su Enciclopedia (Blom, 2007, p. 202):

Aquí son textos hinchados, exorbitantes, allí flacos, pequeños, mezquinos y descarnados. En un lugar parecen esqueletos, en otro dan la impresión de estar inflados: somos, alternativamente, enanos y gigantes, colosos y pigmeos, rectos, bien proporcionados, o jorobados, cojos y mal formados. Añádase a estas grotescas formas un discurso que en ocasiones es abstracto, oscuro o rocambolesco, pero más a menudo resulta descuidado, pasado de rosca y negligente, y cabe compararnos entonces con esos monstruos que aparecen en poesía, si no con algo mucho peor. Pero estos fallos son inherentes a un primer intento... y los siglos venideros los corregirán.

La capacidad de corrección, la tensión permanente entre puntos de vista, nuevas fuentes, o nuevas relaciones, proporciona, si no la calidad inmediata, sí la posibilidad de merodearla, mediante la suma de las actividades intelectuales de diferente rango, intensidad y vocación.

\section{Competencias y cultural digital}

Si bien Wikipedia es una fuente de información que puede ser objeto de estudio en el campo de las Ciencias de la Documentación, en este trabajo adoptamos un enfoque más amplio, desde el componente de "colaboración" presente en la competencia digital e informativa y las prácticas culturales digitales (Sarasa, 2006). En el actual marco europeo de convergencia figura el trabajo colaborativo como competencia interpersonal dentro del proyecto Tuning Educational Structures in Europe (Tuning..., 2000-11).

En conjunción con las habilidades digitales es conveniente presentar brevemente varios conceptos.

Las wikis han sido tratadas ampliamente en la bibliografía sobre docencia universitaria, tanto en la comprensión de su modelo de funcionamiento como en la reseña de experiencias y proyectos. Podemos citar especialmente los dos números monográficos de la Revista de Docencia Universitaria (RED U) sobre "Wiki y educación superior en España” (RED U, 2009). De ellas se destacan algunas ventajas visualización y seguimiento del proceso, especi- 
ficidad de la retroalimentación y reducción de la linealidad en la relación docente-estudiante-, así como elementos críticos a controlar expectativa de corrección, volumen de información, dificultad en la evaluación del esfuerzo y necesidad de que el trabajo no virtual también sea tenido en cuenta (Montenegro y Pujol, 2009).

Otro concepto de indudable aplicación (Mancho, Porto y Valero, 2009), habitualmente presente tras la etiqueta Web 2.0 es el de

[...] crowdsourcing como modelo de producción y resolución de problemas. No queda referido únicamente a la colaboración o al contenido generado por los usuarios, sino a la factibilidad, a través de las tecnologías digitales de conexión, de generar valor y alterar mecanismos de producción asentados. La participación masiva en proyectos colaborativos, conforme a reglas y con un amplio margen de intervención y negociación, permite afrontar proyectos, en nuestro caso, vinculados con la producción social de conocimiento. Los ciclos económicos y de autoridad se ven fuertemente afectados.

Los elementos presentes en la red no los podemos considerar puramente tecnológicos, sino también culturales, característicos de una sociedad (Lara, 2009, p.16)

donde se desarrollan modelos abiertos de información y se valora la capacidad para compartir y construir conocimiento de forma colaborativa. Estas dinámicas propias de esta sociedad globalizada y digital también cuestionan los modelos tradicionales de autoridad y reputación, lo cual tiene consecuencias directas en todos los sectores sociales implicados en la generación y divulgación de conocimiento.

La fluidez de los contenidos digitales tampoco debe hacernos perder de vista que la definición del alcance de los diferentes medios y géneros sigue teniendo importancia a la hora de elegir entre las fuentes de información. La información enciclopédica generalista y especializada sigue cumpliendo un papel de orientación y contextualización, que está en la base de Wikipedia. Dentro de la multiplicidad de opciones de comunicación de Internet, la acumulación ordenada de artículos estructurados adquiere un gran valor en sí misma como punto de referencia frente a la dispersión de canales. En este caso además tiene un doble valor como fuente y como publicación, porque podemos adoptar tanto el rol de usuario como el de productor.

Por último es digno de destacarse el factor movilizador de recursos intelectuales que es la creatividad. La competencia digital no consiste sólo en manejar habilidosamente herramientas, sino también la transformación de la información (adquisición, acceso, evaluación y tratamiento) en procesos de comunicación interpersonal y social, que conducen tanto a la comprensión de las prácticas sociales y culturales de la sociedad del conocimiento como a la posibilidad de ejercer la ciudadanía digital. La creatividad estimula la comprensión de los contenidos docentes, a los cuales se llega de forma indirecta en respuesta a la necesidad surgida de procesos y proyectos. ¿Puede ser Wikipedia una de esas "ocasiones para la creatividad en contextos de educación superior"? Gran parte de los investigadores educativos parecen estar de acuerdo en que los aprendizajes no solo dependen de procesos cognitivos individuales (Elisondo; Donolo y Rinaudo, 2009, p. 6)

[...] sino que las percepciones sobre sí mismo, los demás y los contextos tienen una incidencia marcada en los desempeños académicos y en las posibilidades de modificarlos. Las expectativas, las valoraciones personales y sociales, los intereses y las motivaciones son variables de alta incidencia en los procesos de enseñanza y aprendizaje.

\section{Wikipedia y las universidades}

En la evolución del proyecto Wikipedia existen también estrategias de promoción que tratan de implicar más al ámbito académico, poseedor de las capacidades intelectuales para perfeccionar los contenidos y asegurar la calidad. La Fundación Wikimedia ha puesto en marcha en Estados Unidos una iniciativa piloto - The Public Policy Initiative (Outreach Wiki contributors, 2011a) - para el periodo 2010-2011 (2), tendente a fomentar la reflexión e incorporación de Wikipedia en el trabajo con alumnos de diferentes ciclos, mejorar artículos como parte de currículum y estimular a las figuras conocidas como Wikipedia Ambassadors (cuya traducción al castellano sería "embajadores de Wikipedia") para que actúen como polos de atracción hacia el proyecto. En el fondo de esta iniciativa subyace una estrategia de gestión para producir tanto información como infraestructuras en las que se basen el diseño y el desarrollo de un modelo sostenible a largo plazo para Wikipedia. En este sentido consideramos absolutamente pertinente dicha iniciativa pues todo modelo de negocio o actividad debe ser sometido a renovadas fuerzas tensoras que aseguren su continuidad a largo plazo y su respuesta al cambiante ecosistema de fuentes de información.

The Public Policy Initiative está siendo seguida por veintidós prestigiosas universidades estadounidenses entre las que se encuentran Harvard, Georgetown, Boston o Nueva York; y actualmente se están desarrollando un total de treinta y tres cursos (Wikipedia contributors, 
2011a) coordinados y tutelados por los Wikipedia Ambassadors, los voluntarios que proporcionan apoyo a los profesores para coordinar las iniciativas didácticas relacionadas con Wikipedia en las aulas, al tiempo que incentivan a los alumnos ayudándoles a participar en tales actividades (Outreach Wiki contributors, 2011b). Esta figura tiene dos perfiles: Wikipedia Campus Ambassadors (Embajadores de Wikipedia en el Campus) y Wikipedia Online Ambassadors (Embajadores de Wikipedia en línea). La única diferencia entre ambos es que mientras los primeros asesoran a los nuevos wikipedistas en persona, los segundos mantienen una relación totalmente virtual durante el proceso de consultas (3).

Los embajadores de Wikipedia son, en definitiva, los representantes locales de la comunidad Wikipedia en la universidad y básicamente proporcionan apoyo a los estudiantes sobre las habilidades y potencialidades de Wikipedia; coordinan con los profesores el diseño y la puesta en marcha de las actividades didácticas relacionadas con Wikipedia y organizan acontecimientos lúdicos para promocionar Wikipedia como fuente de información y herramienta docente.

Wikipedia School and university projects (Wikipedia contributors, 2011b) es otra iniciativa que intenta publicitar el esfuerzo colaborativo de enriquecimiento de los contenidos de Wikipedia realizado por docentes y alumnos de algunas escuelas y universidades de todo el planeta. Los coordinadores de esta iniciativa coinciden en enunciar las ventajas que reporta al alumno el uso y mejora de Wikipedia como actividad curricular. Entre otras muchas se podrían citar la mayor asunción de responsabilidad en la ejecución de las tareas asignadas, el incremento de la capacidad crítica a la hora de evaluar las fuentes informativas o el desarrollo de una mayor empatía para trabajar en entornos colaborativos. Además, de esta actividad también se beneficia la comunidad de Wikipedia puesto que aumenta el volumen de conocimiento publicado y se incrementa el nivel de comprensión y fiabilidad de Wikipedia como fuente (Wikipedia contributors, 2010). En la misma línea no podemos dejar de resaltar los Wikipedia student clubs (Wikipedia contributors, 2011c), otra iniciativa que se está desarrollando en algunas universidades y que surge de la iniciativa de los propios alumnos en su empeño por mejorar la herramienta. La actividad, surgida en la Universidad de Michigan, cuenta ya con sucursales en otras universidades estadounidenses y británicas.

Al igual que otros proyectos de participación ciudadana, las políticas abiertas e igualitarias se combinan con acciones de dinamización, tendentes a crear beneficios de reputación, visibilidad y un espacio público de debate, que incorpore el trabajo en Wikipedia a los criterios de valoración social. Ya son muchos los profesores que sugieren la utilización de Wikipedia para la educación. Coincidimos con Guiote (2009) en considerar que el hecho de que puedan existir errores durante el proceso es algo positivo para la enseñanza ya que el cruce de información propicia el acceso y valoración crítica a la información. No obstante, muchos docentes se preguntan en qué momento el esfuerzo dedicado a los proyectos de autoría colectiva y de innovación digital, será tenido en cuenta en el baremo que evalúa el desarrollo de la carrera docente y la reputación académica. Ciertamente, el esfuerzo y el tiempo dedicados deberían tener más allá de la satisfacción personal que reporta el hecho de haber contribuido a generar un enriquecimiento colectivo del conocimiento- una mayor recompensa curricular. Si esto fuera posible, estamos convencidos de que muy probablemente aumentaría el número de docentes implicados en esta labor.

En la reflexión estratégica de las Universidades aparece la tecnología como factor clave tanto para producir como para afrontar el cambio en la función docente, investigadora y cultural. Si ampliamos el foco, desde los portales y sistemas del campus, hacia el entorno social de la información, la presencia en Wikipedia implica estar en uno de los puntos donde se está construyendo una alteración sustancial del paradigma de la comunicación social y científica a través de la cultura de la participación abierta, ya que, como bien afirma O'Sullivan (2009)

cada artículo en Wikipedia, incluso cuando son escritos predominantemente por una única persona, con toda probabilidad, se trata de una copia editada, revisada por expertos, sobre el que se discutió o al que se agregaron imágenes por parte de muchas otras personas. Una cultura basada en compartir y participar es la características más radical de todo el proyecto y lo más prometedor para el futuro de Internet, y por tanto de nuestro modo de vida.

\section{Proyecto de innovación docente "Comunidad de prácticas en Wikipedia"}

A raíz de la convocatoria de proyectos de innovación educativa con TIC (Convocatoria R519/2010) del Vicerrectorado de Relaciones Internacionales e Innovación de la Universidad de Murcia, durante el curso 2010/2011 se ha materializado en la Facultad de Comunicación y Documentación la línea de actividad denominada "TALLER WIKIPEDIA: Comunidad de prácti- 
cas sobre contenidos de Wikipedia". El proyecto se enmarca en la línea de la convocatoria: "Proyectos dirigidos a diseñar e implementar una metodología innovadora en cuanto a la forma de trabajar con los alumnos, teniendo en cuenta los elementos curriculares definidos en la Guía Docente".

Los objetivos específicos planteados han sido:

- Crear una comunidad de práctica sobre contenidos culturales regionales en Wikipedia para alumnos de últimos cursos de las titulaciones de la Facultad.

- Incorporar la reflexión crítica sobre contenidos, enfoques, cobertura de Wikipedia en el ecosistema de los medios sociales.

- Desarrollar habilidades de trabajo digital tanto en el ámbito de pequeños equipos de trabajo para objetivos concretos, como en la totalidad del grupo que abarca a toda la clase.

El proyecto se divide en dos líneas: actividades abiertas a toda la comunidad universitaria y actividades programadas en las asignaturas. El profesorado participante (4) se compromete a incluir una actividad teórica o práctica vinculada a Wikipedia (desde una perspectiva informativa, cultural, comunicativa o técnica) en el marco de una de sus asignaturas de diplomatura, licenciatura, grado o máster, y también a elaborar una ficha resumen de la actividad para la memoria final.

A lo largo del segundo y tercer trimestre del curso se ha creado un grupo de trabajo voluntario abierto a diferentes titulaciones y exalumnos, con el que se ha creado una comunidad de prácticas para editar y reflexionar sobre Wikipedia, introduciéndolos en la edición y completando varios artículos. Este grupo se reunía una vez a la semana, compartiendo las dudas y problemas encontrados, utilizando un aula virtual para compartir materiales y supervisar actividades.

Al mismo tiempo, se trató de ampliar el alcance introduciendo en alguna actividad profesional ponencias relacionadas con la generación de conocimiento colaborativo. En la jornada Contenidos locales digitales, organizada por ANABAD Murcia (Asociación profesional de Archiveros, Bibliotecarios, Documentalistas y Museólogos) y la Biblioteca Regional de Murcia se trató el caso de las enciclopedias locales participativas basadas en wiki, a partir del caso de CTPedia del Ayuntamiento de Cartagena (ANABAD Murcia, 2010; Saorín y Haro, 2011). También se realizó un taller abierto a usuarios de la Red de Bibliotecas Públicas de la ciudad de Murcia, con el título ¡Atrévete con Wikipedia!.
En la misma línea se realizó otro taller orientado al sector de los centros culturales y museos, en el marco de las Jornadas de "Archivos en centros de arte y creación cultural”, organizadas por el Centro Cultural Puertas de Castilla, que se tituló Liberando archivos gráficos: taller Wikimedia Commons. Además se ha impartido un curso reservado a profesorado universitario, con un enfoque práctico de Wikipedia como instrumento para introducir dinámicas participativas y tecnológicas. La acción formativa está recogida en al plan de formación del Centro de Formación y Desarrollo Profesional de la Universidad y se titula Wikipedia en la enseñanza superior: participación, problemáticas y orientaciones didácticas.

Estas actividades han tratado de acercar la dinámica universitaria a otros contextos en los que hay un interés por la comunicación cultural y la participación social. Wikipedia actúa en este caso como espacio compartido entre el conocimiento social y al academia. También se ha creado un blog con actualidad sobre Wikipedia y seguimiento del proyecto denominado "Wikipedia LAB UMU", como punto de información y contacto en línea, con continuidad durante los próximos cursos (Wikipedia LAB UMU, 2010).

\section{Wikipedia como herramienta docente}

Encomendar al estudiante trabajos académicos directamente sobre Wikipedia (Wikipedia assignments) aporta ciertas ventajas frente a otro tipo de informes tradicionales y frente a proyectos wiki de aula. Estas ventajas lo son tanto para el alumno como para el profesor y para la comunidad (Konieczny, 2010).

Por un lado, el estudiante percibe una audiencia real para lo que está haciendo y esto le da más sentido al conjunto de la tarea. Además será consciente de que otros continuarán su trabajo cuando termine la tarea escolar obligada, obteniendo una visión real del proceso de creación de los contenidos de Wikipedia. Esto le permitirá extraer sus propias conclusiones de en qué casos debería usar Wikipedia y en cuáles no.

De este modo, el estudiante asume que no sólo es consumidor de información; puede ayudar a crearla. En el proceso tendrá que adaptarse a exigencias de referenciar cada párrafo y aprender a diferenciar entre fuentes fiables y no fiables.

El profesor contará además con la propia comunidad de editores en el seguimiento de los trabajos de los alumnos, y una serie de procedimientos y utilidades para seguir el historial detectar plagios o violación del copyright. 
La comunidad Wikipedia obtiene un beneficio directo no sólo en el incremento de su contenido: también aumenta el número de personas que acceden a los conocimientos para editar en Wikipedia y que incluso pueden llegar a quedarse como contribuidores estables.

Coincidimos con Peña (2006) acerca de los objetivos en los que puede ayudar Wikipedia, dependiendo de la manera en que ésta se use. Así pues, se podrá estimular a los estudiantes a leer más sobre los temas tratados en el aula y compartir información, al tiempo que se potencia la colaboración mediante el trabajo en grupo.

A lo largo de las distintas actividades realizadas dentro del Proyecto de innovación docente Comunidad de prácticas en Wikipedia han podido detectarse algunas circunstancias dignas de mención al utilizar Wikipedia como herramienta docente. En este sentido, conviene recoger información sobre la percepción de los alumnos antes y después de realizar la actividad para valorar el nivel de dificultad, los elementos motivadores o la proyección de las destrezas desarrolladas en su futuro profesional o académico. El docente deberá tener en cuenta que un trabajo en Wikipedia poco pautado y controlado puede tener efectos no deseados (borrado de esbozos o contenidos no enciclopédicos, vandalismo, plagios, etc.).

Además conviene familiarizar al alumno no sólo con la sintaxis wiki para construir artículos sino con los sistemas de alertas, vigilancia, borrado y negociación.

En este sentido, el grupo de trabajo ha elaborado un decálogo de consejos prácticos para el desarrollo de trabajos académicos por parte de los estudiantes:

- Disponer de cuentas de usuario individuales y nunca de grupo. Tanto el profesor como el grupo conocerá la identidad de cada participante. Es recomendable activar el correo en las cuentas de usuario.

- Registrar el proyecto en el lugar adecuado de Wikipedia: datos de la institución, contexto educativo particular, temas y artículos sobre los que se trabaja, calendario, etc. Esto también implica crear una página Wiki con el programa y planificación de la actividad. En este sentido, deben crearse subpáginas "sandbox" en la zona de usuarios, para experimentar con la composición de artículos y conseguir destreza.

- Comenzar con pequeños trabajos sencillos y útiles, editando un párrafo o sección de artículos, añadiendo bibliografía, retocando pies de fotos o añadiendo una referencia. Esto produce un resultado inmediato beneficioso en el alumno, al ver que un trabajo bien hecho resulta útil de forma inmediata.

- Hay que tener en cuenta que es posible que los estudiantes puedan entrar en conflicto con otros editores de Wikipedia, y que habrá que desarrollar habilidades para mediar y resolver conflictos y fomentar la participación en las páginas de discusión de los artículos. Puede ser conveniente agrupar e identificar en ellas las participaciones de los alumnos del taller. A menudo también surgirán debates sobre el alcance de la propiedad intelectual, el derecho de cita o la reutilización de la información, cuya resolución, conforme a las políticas de Wikipedia, permitirá a los alumnos obtener un conocimiento desde la experiencia directa.

- Antes de escribir un artículo propio, encargar a los alumnos revisar alguno de los artículos, aplicando los criterios de selección de "artículos buenos". La revisión de calidad de los artículos puede realizarse en el aula, como otro aprendizaje más, separada de los procesos propios de Wikipedia.

- Localizar y seleccionar artículos para mejorar o crear también debe contemplarse como una tarea, y por lo tanto debe incluirse en la planificación. Aquí deberán tomarse decisiones sobre la elección del título o la dispersión bajo diferentes entradas, aplicar estructura wiki (wikificar) o añadir recursos de Wikimedia Commons. La selección de artículos en diverso estado de elaboración será clave. Partiendo de artículos con claras necesidades de mejora las tareas de edición inicial serán más eficaces. Contar con artículos en diferentes fases de elaboración también amplia las opciones de trabajo. También es formativo revisar discusiones de borrado de artículos.

- Insistir en aplicar con rigor una política de referencia de hechos, ideas y afirmaciones. Por tanto, la edición de artículos no puede realizarse de forma apresurada, porque requiere informarse sobre el tema en cuestión, al mismo tiempo que resuelven los problemas técnicos del lenguaje wiki. Ha de planificarse el seguimiento y el avance progresivo, evitando errores por acumulación de tareas, siendo conscientes en todo momento de la diferencia entre trabajo original y trabajo enciclopédico: la reflexión libre y la opinión, dispone de otros espacios wiki fuera del marco de Wikipedia 
- Convertir en una tarea del grupo el elevar un artículo hasta la categoría de "artículo bueno" o "destacado" sería un logro evidente, que seguramente no podrá realizarse si los alumnos no cuentan con un bagaje previo. Los plazos de elaboración y, especialmente, los de revisión por la comunidad, han de tenerse en cuenta en el cronograma. Es decir, lograr una motivación más allá del programa académico, incidiendo en la importancia de contar con una experiencia real en el trabajo colaborativo digital como un posible elemento valioso en su currículum.

- Analizar el enfoque de artículos existentes: desde el punto de vista de los contenidos (vinculados a la materia de la asignatura) o desde el punto de vista de las políticas (punto de vista neutral o verificabilidad). En clase se reproduciría la actividad que habitualmente se desarrolla en la página de discusión del artículo.

- Las dinámicas de trabajo más frecuentes son en pequeños grupos, frente al trabajo individual o colectivo de todo el conjunto de estudiantes. Hay que incidir en la necesidad del trabajo en grupo, porque la práctica de cada alumno debe tener en cuenta el funcionamiento abierto y dialéctico de la comunidad (la de la asignatura y la de toda la comunidad Wikipedia). Será interesante que la actividad se enmarque en grupos de interés de campus o temáticos, que puedan servir para continuar de forma voluntaria. Además permitirá contar con apoyos, refuerzos u organizar prácticas compartidas entre asignaturas diferentes de la misma o diferente titulación.

Estas recomendaciones se han recogido a partir de la experiencia y consideraciones que parten de la aceptación de la existencia de Wikipedia y su lugar preponderante en los hábitos de información de muchos estudiantes. Por tanto, consideramos que hay que asumir en Wikipedia un papel activo, limitando de este modo los efectos negativos que puedan plantear sus contenidos al tiempo que se aprovechan para estimular la capacidad de elaboración de información.

\section{Conclusiones}

La motivación de producir valor real quiza sea una de las mayores ventajas de trabajar con Wikipedia desde la Universidad. La calidad del trabajo viene definida por unas normas negociadas por la comunidad que trascienden el marco del aula y que producen un significado en el mundo real. Su utilización requiere, además, destreza y práctica entre el profesorado, la creación de grupos de trabajo en los campus y la iniciación de un proceso de largo recorrido de transformación cultural y de valores. Wikipedia refleja un potencial al que podemos sumarnos para entenderlo. La acción alrededor de los artículos de Wikipedia es un recurso útil para desarrollar las habilidades de escritura formal, el uso de fuentes $y$, de forma combinada, las competencias digitales y de trabajo en grupo.

La conclusión central de este trabajo es la afirmación de que cualquier dinámica educativa que gire alrededor de un uso reflexivo de Wikipedia y de sus contenidos, tiene un alto potencial de mejora de un buen número de capacidades y competencias informacionales de manejo de información, evaluación de fuentes y comprensión del ecosistema digital. Nuestra experiencia, también ha demostrado que Wikipedia constituye una valiosa herramienta para desarrollar competencias relacionadas con el trabajo en equipo, así como con la resolución de conflictos y el logro de acuerdos para elaborar y revisar contenidos a partir de un punto de vista neutral y puesto en común entre los miembros del grupo.

Por otro lado, la organización de actividades concretas, de corta duración (en forma de seminarios, proyectos específicos para mejorar un conjunto definido de artículos o actos sociales lúdico-formativos) puede complementar el trabajo de largo alcance que se desarrolla a lo largo de meses en una asignatura o curso de formación. Eventos del tipo "Wikipedia Party" suponen la elaboración de artículos o contenidos multimedia para su presentación final, permitiendo hacer visible el trabajo de la comunidad de wikipedistas en un campus o facultad.

El uso de Wikipedia como herramienta docente en el ámbito académico universitario se refuerza con la existencia de un grupo de trabajos que perduren en el tiempo, en forma de "laboratorios de ideas" que articulen y coordinen esta actividad a lo largo del tiempo entre diferentes grupos de alumnos, e incluso de diferentes niveles de estudio y ámbitos sociales.

La afirmación anterior se justifica al constatar que, con el paso del tiempo, Wikipedia adquiere mayor resonancia en el inabarcable panorama de la sociedad, la economía y la cultural digital. Lo extraño es la falta de grupos visibles y activos en Wikipedia procedentes del ámbito universitario en España. El espacio abierto e informal está ocupado por otros grupos. ¿Será una ocasión perdida por la Universidad?

Por este motivo hay que trascender un panorama en el que los estudiantes se limiten a usar Wikipedia para consultar información y resolver dudas, ya que cualquier actividad que les acer- 
que a un conocimiento más profundo o a una visión crítica de la misma, supondrá una mayor capacidad para la elaboración del trabajo intelectual.

\section{Notas}

(1) Wikimedia Commons (frecuentemente abreviado como Commons) es un proyecto de la Fundación Wikimedia cuyo objetivo es servir como almacén de archivos de imágenes y otros formatos multimedia para el resto de los proyectos de la fundación Wikimedia de todos los idiomas.

(2) La misma iniciativa se repetirá en India en el periodo 2011-2012.

(3) En la actual edición hay 54 Wikipedia Campus Ambassadors y 91 Wikipedia On line Ambassadors.

(4) En su diseño y realización participan los siguientes profesores del Departamento de Información y Documentación: Tomás Saorín, Francisco Javier Martínez Méndez, José Antonio Gómez, Juan Antonio Pastor, Rosana López y María Verónica de Haro.

\section{Referencias}

(RED U: Revista de Docencia Universitaria. Números extraordinarios 4 y 5 sobre "Wiki y educación superior en España (I y II) de 2009. http://redaberta.usc.es/redu/in dex.php/REDU

ANABAD Murcia (2010). Jornada profesional Contenidos digitales locales: modelos institucionales y participativos. Murcia: ANABAD, 2010. http://www.anabadmurcia.org/ $? p=3$

Assouline, P. (2008) Y dicen que es una fuente. // Gourdain, P.; Martínez Soliman, M. (eds.). La revolución Wikipedia. Madrid: Alianza, 2008.

Barberà, E. (2009). Filosofía wiki: el compromiso de las soluciones. // Revista De Docencia Universitaria. 7:3. http://redaberta.usc.es/redu/index.php/REDU/article/view /100 (4-2-2011).

Blom, P. (2007). Encyclopédie: El triunfo de la razón en tiempos irracionales. Madrid: Anagrama, 2007.

Broughton, J. (2008). Wikipedia: The Missing Manual. O'Reilly Media, 2008.

Elisondo, R.; Donolo, D.; Rinaudo, M. (2009). Ocasiones para la creatividad en contextos de educación superior. // Revista De Docencia Universitaria. 7:4. http://reda berta.usc.es/redu/index.php/REDU/article/view/112 (4-22011).

Gómez-Hernández, J. A.; Saorín Pérez, T. (2011). Wikipedias y biblioteca pública: Participar en la información local digital a través de "localpedias". // Anuario ThinkEPI. $5,78-84$.

Guiote, A. (2009). La creación colectiva de mensajes informativos en la web 2.0: el caso Wikipedia. Fundación Europea para la Sociedad de al Información y la Administración Electrónica, Euroeditions, 2009.

Head, A. J.; Eisenberg, M.B. How College Students Evaluate and Use Information in the Digital Age. Project Information Literacy Progress Report, nov. 2010.
http://projectinfolit.org/pdfs/PIL Fall2010 Survey FullRe port1.pdf (12-03-2011).

Konieczny, P. (2010). Teaching with Wikipedia and other Wikimedia foundation wikis. // Proceedings of the 6th International Sym-posium on Wikis and Open Collaboration, WikiSym '10, article 29. New York: ACM, 2010. DOI=10.1145/1832772.1832810.

http://doi.acm.org/10.1145/1832772.1832810 (2-4-2011).

Lara, T. (2009). El papel de la Universidad en la construcción de su identidad digital. // RUSC. 6:1. http://www. uoc.edu/ojs/index.php/rusc/article/viewFile/25/19 (5-42011).

Lih, A. (2009). The Wikipedia Revolution: How A Bunch of Nobodies Created The World's Greatest Encyclopedia. New York: Hype-rion, 2009.

Mancho Barés, G.; Porto Requejo, M.; Valero Garcés, C. (2009). Wikis e innovación docente. // Revista De Docencia Universitaria. 7:3. http://redaberta.usc.es/redu/ index.php/REDU/article/view/101 (4-2-2011).

Montenegro, M.; Pujol, J.(2009). Evaluación de la wiki como herramienta de trabajo colaborativo en la docencia universitaria. // Revista de Educación a Distancia. http://redaberta.usc.es/redu/index.php/REDU/article/view /104 (13-5-2011).

O'Sullivan, D. Wikipedia, the new community of practice. Burlington, VT: Ashgate, 2009.

Outreach Wiki contributors (2011a). Public Policy Initiative. // Outreach Wiki. Wikimedia Fondation. http://outreach.wi kimedia.org/wiki/Public_Policy_Initiative

Outreach Wiki contributors (2011b). Wikipedia Ambassador Program. // Outreach Wiki. Wikimedia Fondation. http://outreach.wikimedia.org/wiki/Wikipedia_Ambassado r_Program

Peña, I.; Córcoles, C.P.; Casado, C. (2006). El Profesor 2.0: docencia e investigación desde la Red. // UOC Papers. 3. http://www.uoc.edu/uocpapers/3/dt/esp/pena_corcoles _casado.pdf (13-5-2011).

Saorín Pérez, T.; de Haro de Dan Mateo, M ${ }^{a}$ V. (2011). Contenidos Digitales Locales. Madrid: Anabad, 2011.

Sarasa Cabezuelo, Antonio (2006). Usando la Wikipedia como motivación en el proceso de aprendizaje. // Revista Latinoamericana de Tecnología Educativa. 5:2, 433442.

Tuning: educational structures in Europe (2000-11). http://www.unideusto.org/tuningeu/

Wikipedia contributors (2010). Category:Wikipedia articles as assignments. Wikipedia Foundation, 2010. http://en.wikipedia.org/wiki/Category:Wikipedia_articles_ as_assignments

Wikipedia contributors (2011a). Wikipedia:WikiProject United States Public Policy/Courses. / Wikipedia. Wikipedia Foundation. http://en.wikipedia.org/wiki/Wikipedia: WikiProject_United_States_Public_Policy/Courses

Wikipedia contributors (2011b). Wikipedia: School and university projects. Wikipedia Foundation, 2010. http://en.wikipedia.org/wiki/Wikipedia:School_and_univer sity_projects

Wikipedia contributors (2011c). Wikipedia student clubs. // Wikipedia. Wikimedia Foundation, 2011. http://out reach.wikimedia.org/wiki/Wikipedia_student_clubs.

Wikipedia LAB UMU (2010-). http://www.um.es/gtiweb/ wikipedialab/. 
